\title{
Characterization of Bacillus subtilis Colony Biofilms via Mass Spectrometry and Fluorescence Imaging
}

\author{
Tong $\mathrm{Si},{ }^{1, \ddagger}$ Bin $\mathrm{Li},{ }^{2,3, \ddagger}$ Ke Zhang, ${ }^{2}$ Yiran $\mathrm{Xu},{ }^{4}$ Huimin Zhao, ${ }^{1,2,4,5, *}$ \\ and Jonathan V. Sweedler ${ }^{2,3,5, *}$ \\ ${ }^{1}$ Department of Chemical and Biomolecular Engineering and Carl R. Woese Institute for \\ Genomic Biology, University of Illinois at Urbana-Champaign, Urbana, IL, 61801, USA \\ ${ }^{2}$ Department of Chemistry, University of Illinois at Urbana-Champaign, Urbana, IL, 61801, USA \\ ${ }^{3}$ Beckman Institute for Advanced Science and Technology, University of Illinois at Urbana- \\ Champaign, Urbana, IL, 61801, USA \\ ${ }^{4}$ Department of Biochemistry, University of Illinois at Urbana-Champaign, Urbana, IL, 61801, \\ USA \\ ${ }^{5}$ Department of Bioengineering, University of Illinois at Urbana-Champaign, Urbana, IL, 61801, \\ USA
}

\section{SUPPORTING INFORMATION}

\section{Contents}

Additional methods.

Figure S1. Colony morphology of B. subtilis strains on MSgg and ISP2 media.

Figure S2. Representative MALDI LIFT ToF/ToF mass spectra of SKF and SDP.

Figure S3. Cell growth of the B. subtilis NCIB3610 strain and its mutants.

Table S1. Strains used in this study.

Table S2. Selected metabolites assigned in B. subtilis biofilms by MALDI ToF MS analysis of intact cells.

\section{Supporting references.}




\section{ADDITIONAL METHODS}

\section{Intact-cell MALDI-MS and LIFT TOF/TOF}

After cultivation, a square section $(7 \mathrm{~mm} \times 7 \mathrm{~mm}$ ) containing the biofilms of the wild type $B$. subtilis NCIB3610 strain was transferred to a $1.7 \mathrm{~mL}$ Eppendorf tube and suspended in $500 \mu \mathrm{L}$ of acetonitrile (ACN)-water solution containing $0.1 \%$ trifluoroacetic acid (TFA) (1:1, v/v). The suspension was further subjected to a $15 \mathrm{~min}$ sonication. Cell residues were separated by centrifugation (5 min, $10,000 \mathrm{rpm}, 4{ }^{\circ} \mathrm{C}$ ), and the supernatant was transferred to a $1.7 \mathrm{~mL}$ Eppendorf tube and dried under vacuum in a rotary flash evaporator (MiVac, GeneVac, UK). The samples were reconstituted in $100 \mu \mathrm{L}$ of ACN-water solution containing $0.1 \%$ TFA (1:1, $\mathrm{v} / \mathrm{v}$ ). For MALDI MS analysis, $1 \mu \mathrm{L}$ of the extracts was mixed with $2 \mu \mathrm{L}$ of DHB solution (50 $\mathrm{mg} \mathrm{mL}^{-1}$ in ACN-water with 0.1\% TFA, 7:3, v/v) and spotted onto a MTP 384 polished steel target (Bruker Daltonics, Billerica, MA). MALDI LIFT TOF/TOF mass spectra were obtained in positive reflection mode on the same MALDI mass spectrometer described in the main text. For each spot, 1000 laser shots fired at $1000 \mathrm{~Hz}$ were collected. The characteristic fragments of those ions and tandem MS spectra of SKF and SDP are shown in Table S2 and Figure S2.

\section{Cell Growth Measurement}

Cell growth was monitored by measuring optical density at a wavelength of $600 \mathrm{~nm}\left(\mathrm{OD}_{600}\right)$ of cell culture using a NanoDrop 2000c Spectrophotometer (Thermo Fisher Scientific, Waltham, MA). Three freshly streaked B. subtilis colonies (biological triplicates) on a luria broth (LB) agar plate were inoculated separately into $3 \mathrm{~mL}$ of LB liquid media for cultivation at $37{ }^{\circ} \mathrm{C}$ and 250 rpm. After $20 \mathrm{~h}$, cells were re-inoculated into $3 \mathrm{~mL}$ of fresh LB liquid media, and initial cell density was adjusted to $\mathrm{OD}_{600}=0.2$ for each culture. Cell density was then measured at indicated time intervals. After $12 \mathrm{~h}, 1 \mu \mathrm{L}$ of cell culture from each replicate was spotted onto MSgg plates supplemented with 1.5\% agar and allowed to grow under static conditions at $37{ }^{\circ} \mathrm{C}$ for $24 \mathrm{~h}$. Colony biofilms were harvested from the MSgg agar surface and placed in $1 \mathrm{~mL}$ of PBS. Biofilms were dispersed with 12 pulses of mild sonication with $1 \mathrm{~s}$ duration and $30 \%$ amplitude on a Fisher Scientific Sonic Dismembrator Model 500. The cell suspension was then subjected to $\mathrm{OD}_{600}$ measurement. For colony biofilms, cell growth was calculated as cell density equivalents, assuming biofilms were suspended as single cells in $1 \mathrm{~mL}$ of liquid culture. 


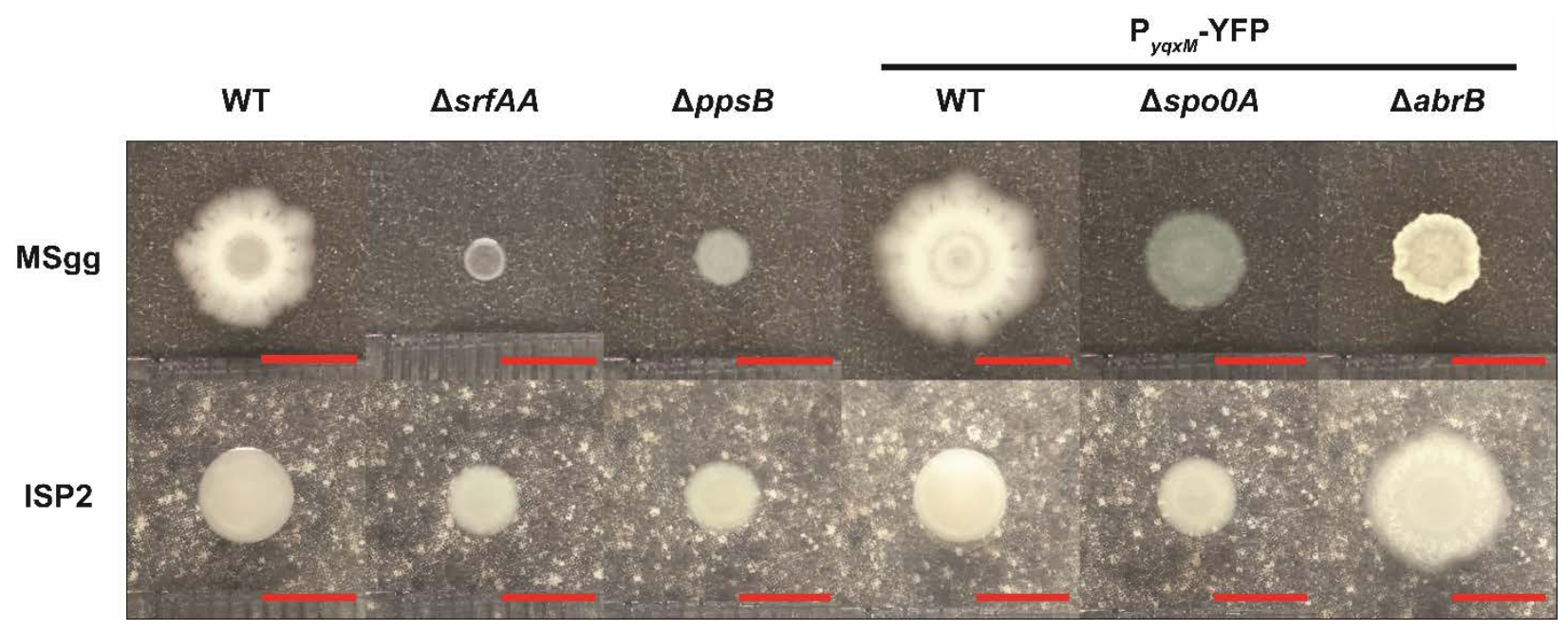

Figure S1. Colony morphology of $B$. subtilis strains on MSgg and ISP2 media. Pictures were taken after incubation at $37^{\circ} \mathrm{C}$ for $24 \mathrm{~h}$ on solid agar media. Scale bars $=5 \mathrm{~mm}$. 


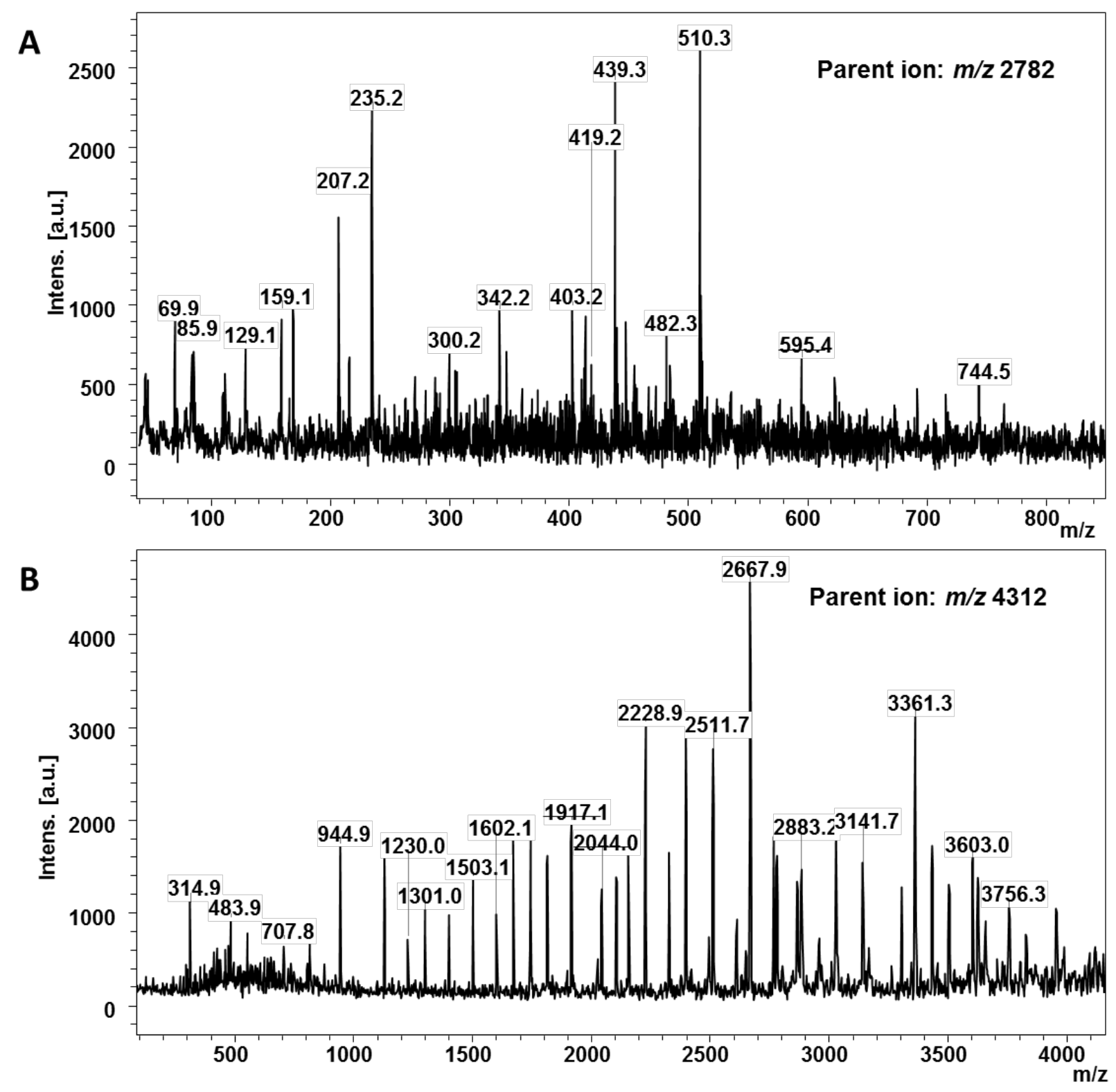

Figure S2. Representative MALDI LIFT TOF/TOF mass spectra of (A) SKF and (B) SDP. 
A

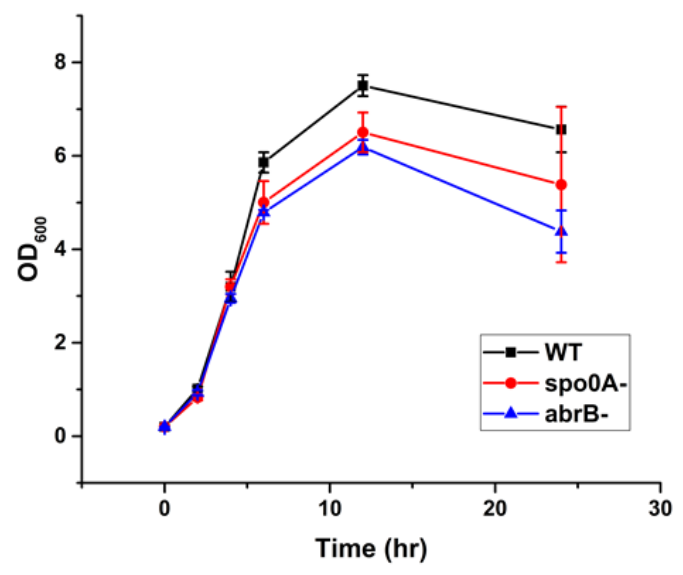

B

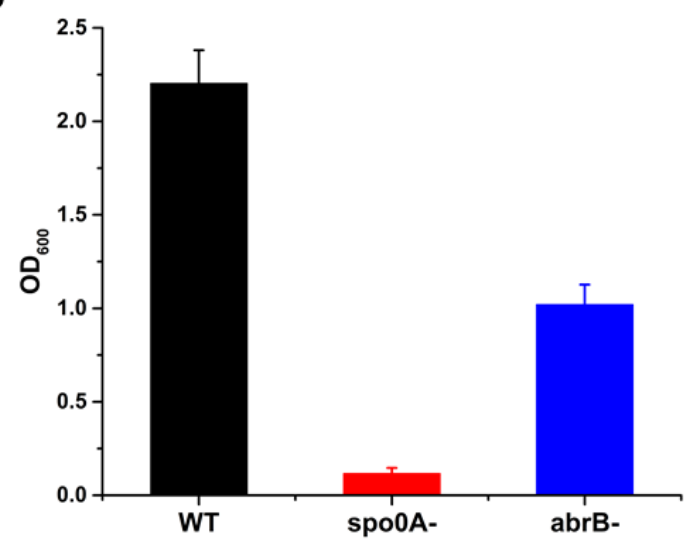

Figure S3. Cell growth of the B. subtilis NCIB3610 strain and its mutants. (A) B. subtilis strains were cultivated in LB liquid media with an initial cell density of $\mathrm{OD}_{600}=0.2$. (B) $B$. subtilis biofilms were developed from $1 \mu \mathrm{L}$ of overnight cell cultures spotted on MSgg agar. After $24 \mathrm{~h}$, biofilms were harvested and dispersed in $1 \mathrm{~mL}$ of PBS using sonication before cell density measurement. For biofilms, the equivalent values of initial cell density were estimated as $\mathrm{OD}_{600}=0.0075 \pm 0.0002(\mathrm{WT}), 0.0065 \pm 0.0004(\Delta s p o 0 A)$, and $0.0062 \pm 0.0002(\Delta a b r B)$, assuming that $1 \mu \mathrm{L}$ of the cell cultures were added in $1 \mathrm{~mL}$ of liquid media. Error bars indicate the SDs of three biological replicates. $P$ values were calculated using the independent two-tailed, two-sample $t$-test for equal sample sizes and equal variance: (A) 0.023 (spo0A-/WT) and 0.0011 (abrB-/WT) at $12 \mathrm{~h}$ for liquid media when cell cultures were spotted onto MSgg agar media; and (B) $3.6 \times 10^{-5}\left(\right.$ spo0A-/WT) and $5.8 \times 10^{-4}(\mathrm{abrB}-/ \mathrm{WT})$ at $24 \mathrm{~h}$ for agar media. 
Table S1. Strains used in this study.

\begin{tabular}{|c|c|c|}
\hline Strain name & Genotype & Sources \\
\hline \multicolumn{3}{|c|}{ Donor laboratory strains } \\
\hline $1 \mathrm{~S} 143$ & PY79 spo0A::kan & BGSC* \\
\hline 1A935 & $168 \mathrm{Cm}$ pheA1 trpC2 abrB::cat & BGSC \\
\hline BK03480 & 168 trpC2 srfAA::erm & BGSC \\
\hline BK18330 & 168 trpC2 ppsB::erm & BGSC \\
\hline \multicolumn{3}{|l|}{ NCIB3610 } \\
\hline $3 \mathrm{~A} 1$ & Wild type isolate & BGSC \\
\hline $3 \mathrm{Al} \Delta \mathrm{srfAA}$ & 3A1 srfAA::erm & This study \\
\hline $3 \mathrm{~A} 1 \_\Delta \mathrm{ppsB}$ & 3A1 ppsB::erm & This study \\
\hline \multicolumn{3}{|c|}{ Reporter strain (ZK3779) } \\
\hline ZK3779_WT & $\begin{array}{l}\text { NCIB3610 amyE:: } P_{\text {srfaA }}-y f p^{* *} \text { (spec), lacA::P } P_{\text {yqxM }} \text {-cfp } \\
\text { (mls) }\end{array}$ & $\operatorname{Ref}^{1}$ \\
\hline ZK3779__spo0A & ZK3779_WT spo0A::kan & This study \\
\hline ZK3779_AabrB & ZK3779_WT abrB::cat & This study \\
\hline \multicolumn{2}{|l|}{ SPP1 phage } & BGSC \\
\hline
\end{tabular}

*BGSC: Bacillus Genetic Stock Center, Columbus, OH

**As only a tiny fraction of the cell population was actively expressed from the $\mathrm{P}_{\text {srfaA }}$ promoter ${ }^{1}$, the YFP fluorescence was too weak for the fluorescence stereoscope, and therefore not studied in this work. 
Table S2. Selected metabolites assigned in B. subtilis biofilms by MALDI TOF MS analysis of intact cells.

\begin{tabular}{|c|c|c|c|c|c|c|}
\hline Compounds & $\begin{array}{l}\text { Molecular } \\
\text { formula }\end{array}$ & $\begin{array}{c}\text { Measured }[\mathrm{M}+\mathrm{H}]+ \\
\text { [theoretical monoisotopic } \\
\text { mass, mass error }(\mathrm{ppm})]\end{array}$ & $\begin{array}{c}\text { Measured }[\mathrm{M}+\mathrm{Na}]+ \\
\text { [theoretical monoisotopic mass, } \\
\text { mass error }(\mathrm{ppm})]\end{array}$ & $\begin{array}{c}\text { Measured }[\mathrm{M}+\mathrm{K}]+ \\
\text { [theoretical monoisotopic mass, } \\
\text { mass error }(\mathrm{ppm})]\end{array}$ & $\begin{array}{c}\text { Fragments } \\
\text { (parent ions, }([\mathrm{M}+\mathrm{Na}]+)\end{array}$ & Ref \\
\hline surfactin $\mathrm{C}_{13}$ & $\mathrm{C}_{51} \mathrm{H}_{89} \mathrm{~N}_{7} \mathrm{O}_{13}$ & ND & $1030.6387[1030.6411,-2.3]$ & ND & ND & $\operatorname{Ref}^{2}$ \\
\hline surfactin $\mathrm{C}_{14}$ & $\mathrm{C}_{52} \mathrm{H}_{91} \mathrm{~N}_{7 \mathrm{O}_{13}}$ & ND & $1044.6540[1044.6567,-2.6]$ & ND & $931.5,800.3,707.3,594.2$ & $\operatorname{Ref}^{2}$ \\
\hline surfactin $\mathrm{C}_{15}$ & $\mathrm{C}_{53} \mathrm{H}_{93} \mathrm{~N}_{7} \mathrm{O}_{13}$ & ND & $1058.6720[1058.6724,-0.4]$ & $1074.6487[1074.6463,2.2]$ & $945.6,814.4,707.3,594.2$ & $\operatorname{Ref}^{2}$ \\
\hline Plipastatin- $\mathrm{C}_{16}$-Ala & $\mathrm{C}_{72} \mathrm{H}_{110} \mathrm{~N}_{12} \mathrm{O}_{20}$ & $1463.7903[1463.8032,-8.8]$ & $1485.7707[1485.7852,-9.8]$ & ND & ND & $\operatorname{Ref}^{2}$ \\
\hline Plipastatin- $\mathrm{C}_{17}$-Ala & $\mathrm{C}_{73} \mathrm{H}_{112} \mathrm{~N}_{12} \mathrm{O}_{20}$ & $1477.7988[1477.8189,-13.6]$ & $1499.7962[1499.8008,-3.1]$ & ND & $1130.9,1016.7$ & $\operatorname{Ref}^{2}$ \\
\hline Plipastatin- $\mathrm{C}_{16}$-Val & $\mathrm{C}_{74} \mathrm{H}_{114} \mathrm{~N}_{12} \mathrm{O}_{20}$ & $1491.8203[1491.8345,-9.5]$ & $1513.8152[1513.8165,-0.9]$ & ND & $1301.6,1130.3,1016.1$ & $\operatorname{Ref}^{2}$ \\
\hline Plipastatin-C $_{17}$-Val & $\mathrm{C}_{75} \mathrm{H}_{116} \mathrm{~N}_{12} \mathrm{O}_{20}$ & $1505.8384[1505.8502,-7.8]$ & $1527.8301[1527.8321,-1.3]$ & $1543.8150[1543.8060,5.8]$ & $1301.6,1130.4,1016.2$ & $\operatorname{Ref}^{2}$ \\
\hline Subtilosin & $\mathrm{C}_{152} \mathrm{H}_{226} \mathrm{~N}_{38} \mathrm{O}_{45} \mathrm{~S}_{3}$ & $3400.5076[3400.5799,-21.2]$ & $3422.5111[3422.5619,-14.8]$ & 3438. $4739[3438.5358,-18.0]$ & ND & $\operatorname{Ref}^{2,3}$ \\
\hline SKF & $\mathrm{C}_{119} \mathrm{H}_{192} \mathrm{~N}_{36} \mathrm{O}_{29} \mathrm{~S}_{6}$ & $2782.2572[2782.3053,-17.3]$ & ND & ND & * & $\operatorname{Ref}^{4}$ \\
\hline SDP & $\mathrm{C}_{200} \mathrm{H}_{307} \mathrm{~N}_{47} \mathrm{O}_{55} \mathrm{~S}_{2}$ & $4312.1293[4312.2185,-20.7]$ & $4334.1719[4334.2004,-6.6]$ & $4350.0520[4350.1744,-28.1]$ & * & $\operatorname{Ref}^{4}$ \\
\hline
\end{tabular}

ND: not detectable

*: Characteristic fragments of ions at m/z 2782 and $m / z 4312$ were shown in Figure S2. 


\section{Supporting References}

(1) Lopez, D.; Vlamakis, H.; Losick, R. Kolter, R. Paracrine signaling in a bacterium. Genes Dev. 2009, 23, (14), 1631-1638.

(2) Yang, Y. L.; Xu, Y.; Straight, P. Dorrestein, P. C. Translating metabolic exchange with imaging mass spectrometry. Nat. Chem. Biol. 2009, 5, (12), 885-887.

(3) Marx, R.; Stein, T.; Entian, K. D. Glaser, S. J. Structure of the Bacillus subtilis peptide antibiotic subtilosin A determined by $1 \mathrm{H}-\mathrm{NMR}$ and matrix assisted laser desorption/ionization time-of-flight mass spectrometry. J. Protein Chem. 2001, 20, (6), 501-506.

(4) Liu, W. T.; Yang, Y. L.; Xu, Y.; Lamsa, A.; Haste, N. M.; Yang, J. Y.; Ng, J.; Gonzalez, D.; Ellermeier, C. D.; Straight, P. D.; Pevzner, P. A.; Pogliano, J.; Nizet, V.; Pogliano, K. Dorrestein, P. C. Imaging mass spectrometry of intraspecies metabolic exchange revealed the cannibalistic factors of Bacillus subtilis. Proc. Natl. Acad. Sci. USA 2010, 107, (37), 16286-16290. 